\title{
Object-based attention: Strength of object representation and attentional guidance
}

\author{
SARAH SHOMSTEIN \\ George Washington University, Washington, D.C. \\ AND \\ Marlene Behrmann \\ Carnegie Mellon University, Pittsburgh, Pennsylvania
}

\begin{abstract}
Two or more features belonging to a single object are identified more quickly and more accurately than are features belonging to different objects - a finding attributed to sensory enhancement of all features belonging to an attended or selected object. However, several recent studies have suggested that this "single-object advantage" may be a product of probabilistic and configural strategic prioritizations rather than of object-based perceptual enhancement per se, challenging the underlying mechanism that is thought to give rise to object-based attention. In the present article, we further explore constraints on the mechanisms of object-based selection by examining the contribution of the strength of object representations to the single-object advantage. We manipulated factors such as exposure duration (i.e., preview time) and salience of configuration (i.e., objects). Varying preview time changes the magnitude of the object-based effect, so that if there is ample time to establish an object representation (i.e., preview time of 1,000 $\mathrm{msec}$ ), then both probability and configuration (i.e., objects) guide attentional selection. If, however, insufficient time is provided to establish a robust object-based representation, then only probabilities guide attentional selection. Interestingly, at a short preview time of $200 \mathrm{msec}$, when the two objects were sufficiently different from each other (i.e., different colors), both configuration and probability guided attention selection. These results suggest that object-based effects can be explained both in terms of strength of object representations (established at longer exposure durations and by pictorial cues) and probabilistic contingencies in the visual environment.
\end{abstract}

Research has established that attentional guidance during visual processing is mediated by spatial locations; target detection at a cued location in space is better than detection at other, uncued locations (Downing \& Pinker, 1985; Eriksen \& St. James, 1986; Posner, Snyder, \& Davidson, 1980). Over the past two decades, however, considerable evidence has accumulated suggesting that attentional selection can also be mediated by objects that are present in the environment. This latter perspective gained ground after several studies demonstrated a benefit that was associated with selecting features of an attended object as compared with features of an unattended object, even when the two relevant objects were superimposed spatially (Duncan, 1984; Kahneman \& Henik, 1981; Rock \& Guttman, 1981). Research has suggested that both objects and spatial locations influence attentional selection.

A large body of evidence in support of object-based attentional selection has been derived from a paradigm that was originally developed by Egly, Driver, and Rafal (1994) and referred to as the two-rectangle method (Marrara \& Moore, 2003). In this paradigm, two adjacent rect- angles - oriented either vertically or horizontally - are presented to the observer. After a brief delay, one end of one of the rectangles is illuminated briefly - an event that cues the observer to direct attention to a specific location while maintaining fixation at the center of the display. After another brief delay, a target is presented in the location previously occupied by the cue (a validly cued location), in the opposite end of the cued rectangle (an invalid same-object location), or in the other rectangle (an invalid different-object location) at the same distance from the cue as the invalid same-object location. This paradigm yields two main findings: Items in the validly cued location are detected faster and more accurately than are items presented in any other location. This result reflects the fact that spatial distance between the cued location and the target affects the quality of the perceptual representation (Desimone \& Duncan, 1995; Müller, Bartelt, Donner, Villringer, \& Brandt, 2003; Posner et al., 1980), and it is consistent with spatial attentional-cuing findings to date. More relevant for the present purpose is the finding that items in the invalid same-object location are

S. Shomstein, shom@gwu.edu 
detected faster and more accurately than those in the invalid different-object location. This latter finding reflects the contribution of object-based attention to the quality of perception (Egly et al., 1994; Müller \& Kleinschmidt, 2003) and indicates that other dimensions of the objects are facilitated by virtue of being part of the cued object. This paradigm has been extended in several subsequent studies investigating the role of object-based attention in visual perception (Behrmann, Zemel, \& Mozer, 1998; Moore, Yantis, \& Vaughan, 1988; Müller \& Kleinschmidt, 2003; Shomstein \& Behrmann, 2006; Shomstein \& Yantis, 2004; Watson \& Kramer, 1999), and the findings are robust and replicable.

Having definitively established the presence of objectbased attentional selection, most recent research on this topic has begun to focus on elucidating the mechanisms that give rise to this object-based advantage (Avrahami, 1999; Cepeda \& Kramer, 1999; Müller \& Kleinschmidt, 2003; Shomstein \& Yantis, 2002, 2004). One such study (Shomstein \& Yantis, 2002) proposed two possible mechanisms that might give rise to an object-based advantage. The first mechanism entails sensory enhancement of the locations within the attended object. This mechanism is in agreement with the competition model originally proposed by Desimone and Duncan (1995) that implies that cortical representations of items within the scene are mutually inhibitory; thus, the strength of a representation is weaker when an object is presented along with other items or when it appears in isolation (i.e., without competition). The object-based advantage emerges because an attentional cue that summons spatial attention to a particular location serves as a top-down biasing signal, guides attention, and strengthens the sensory representation at the attended location or object. The enhancement afforded to the attended locations allows it to compete more effectively with other representations (Kastner, De Weerd, Desimone, \& Ungerleider, 1998). Given the enhanced activation for a part of the object, attention "spreads" to other features or locations that are bound by the object (Corbetta, Miezin, Dobmeyer, Shulman, \& Petersen, 1990; O'Craven, Downing, \& Kanwisher, 1999), reflecting object-based "sensory enhancement."

The second mechanism, proposed by Shomstein and Yantis $(2002,2004)$, is the attentional prioritization view, in which the single object advantage emerges from the order in which different regions of the scene are visually inspected when there is uncertainty about the target location in the display. We term this type of attentional guidance configural prioritization. The mechanism proposed is based on the supposition that-following complex inputs into the visual system, and ceteris paribus - the organism learns to assign higher attentional priority to locations of high importance (e.g., cued locations) and to locations within an already-cued object rather than to locations elsewhere. Given that attentional priority determines the order in which the visual scene will be investigated, unattended parts of an attended object will enjoy an attentional advantage over other objects and locations in a scene (Müller \& Kleinschmidt, 2003). It is useful for one to view this account in the context of Wolfe's (1994) guided search, which suggests that the order of search is governed by attentional priorities that are affected by not only local feature contrast and similarity to a target template (Cave \& Wolfe, 1990; Wolfe, 1994), but also by object structure.

At a first glance, configural prioritization looks very much like sensory enhancement. In fact, in the two-rectangle paradigm, the two types of mechanisms predict an identical effect of invalid same-object targets being detected faster than the invalid different-object targets, and both being slower than validly cued targets. However, Shomstein and Yantis (2002) demonstrated that under the circumstances, when the contribution of each mechanism could be dissociated, the pattern of results obeyed that of configural attentional prioritization rather than that of sensory enhancement (see Shomstein \& Yantis, 2002, for details of that study).

Note that the object-based advantage observed in the two-rectangle method could also be explained by yet another prioritization strategy - probabilistic guidance. Unlike configural prioritization, this strategy predicts that the object-based advantage is largely driven by probabilistic imbalances that are present within the visual scene. Egly et al. (1994) used $75 \%$ cue validity, with the remaining $25 \%$ of the trials invalidly cued and split evenly between same and different object locations (i.e., 12.5\% for each). As a result of this probabilistic arrangement, it follows that targets appeared within a cued object (i.e., the valid and the invalid-same object locations) on $87.5 \%$ of the trials. Therefore, simply on the basis of the probability of target occurrences, items within the cued object-either the validly cued location or the invalid within-object location - might be assigned high priority.

Of course, the finding that probability and configural prioritizations guide attentional selection is not new in and of itself, and research conducted over the past 30 years has demonstrated that target-location probabilities can influence the way in which a visual display is searched (see, e.g., Chun \& Jiang, 1998; Geng \& Behrmann, 2002; Jiang \& Chun, 2001; Miller, 1988; Moore \& Egeth, 1998; Shaw, 1978). What is novel is the suggestion by Shomstein and Yantis (2004) that the object-based advantage in the two-rectangle method might be explained simply by these probabilistic (statistical) imbalances and by configural prioritizations without appealing to a sensoryenhancement model of object-based attention. On the basis of these findings, researchers concluded that the two types of attentional prioritizations - configural and probabilistic - and not the early sensory enhancement, may suffice to give rise to object-based advantage.

In order to understand the relative contribution of configural and probabilistic prioritizations to the emergence of the object-based advantage, Shomstein and Yantis (2004) modified the two-rectangle paradigm so that the two types of prioritizations (configural and probabilistic) made different predictions regarding the pattern of the reaction times (RTs). The two-rectangle method was used with the cue validity of $50 \%$. Attentional priority was manipulated by varying the probability that a target would appear in each of the two uncued locations. On invalidly cued trials, the target appeared in the high-probability location (defined by an absolute spatial location, e.g., upper right) 
$83 \%$ of the time and in the low-probability location (e.g., lower left) $17 \%$ of the time. In both conditions, targets appeared in the cued object on half of the trials, and in the uncued object on the other half of the trials. In addition, stimulus onset asynchrony (time between the cue and the target, SOA) was varied between $200 \mathrm{msec}$ and $600 \mathrm{msec}$ in order to determine whether the relative contributions of the two sources of priority (configural and probability based) differ over time (i.e., compete over time for control over priority assignment). The prediction was as follows: If probabilities alone are guiding the search, then RTs should be the fastest at the cued location $(50 \%)$ and faster at the high-probability (43\%) than at the low-probability (7\%) location, independent of whether the target appears in the cued or uncued object (i.e., only probabilities predict RTs). If, on the other hand, object configuration alone determines RT responses, then RTs should be the fastest at the cued location and faster at the invalidly cued sameobject than at the invalidly-cued different-object location, independent of whether the target appears in the high- or low-probability location. And, of course, if both object configuration and probability guides attentional search, then additive or interactive effects of these two factors should be observed. The authors observed that at a short SOA $(200 \mathrm{msec})$, the object configuration and probability effects were approximately additive. However, at a longer SOA (600 msec), the object configuration effect disappeared, and RTs depended exclusively on rank ordering of probability. The authors concluded that both types of prioritization - configural and probability based - guide attentional selection. Furthermore, they suggested that configural prioritization is the default attentional mechanism; object-based effects are observed even in the presence of a more predictive and effective way to search the scene (i.e., on the basis of local probability contingency alone). However, the default can be overridden by a more effective strategy if the SOA is increased and, as such, there is more time to suppress this default setting.

In the present article, we explore the contribution of exposure time and the strength of object representation on probabilistic and configural contributions to object-based effects. For example, an immediate and outstanding question concerns the relative contribution of time (i.e., object exposure duration) to the adoption of probability-based and configural prioritizations. Shomstein and Yantis (2004) suggested that at short SOAs - at which both object-based and probability effects were observed - configural prioritization is a default setting of the system. However, as the time increases between the cue and the target, this default setting is suppressed, and only probabilities guide performance. What remains unclear from these findingsand is, in fact, a confound - is whether the crucial factor that predicts the abandoning of configural prioritization is the duration of SOA or the absolute exposure duration (i.e., the total time that objects were presented to the subjects $-1,200 \mathrm{msec}$ for the SOA 200 -msec condition and $1,600 \mathrm{msec}$ for the SOA 600 -msec condition). Longer exposure to objects allows more time for perceptual grouping and figure-ground segmentation, as well as longer "experience" with the objects. Another possibility is that absolute exposure duration is not predictive of which prioritization will be guiding attentional selection, but of the exposure before and after the cue onset becomes an important factor. A short preview time might not be sufficient to establish strong-enough object representations, leaving insufficient time for object-based effects to manifest.

A second issue that deserves attention is the nature of the contribution by configural representations and why the effects of this representation diminish with long SOAs. One possibility is that the "objects" used in earlier studies were rather weak (open rectangles defined by black border); thus, the activation decayed rather quickly. One might ask, therefore, whether a stronger cue to objecthood (i.e., two colored surfaces) might bias the configural objectbased guidance so that object-based effects might persist even with long SOAs.

In order to manipulate both the timing and the strength of the object representations, we adopted the Shomstein and Yantis (2004) two-rectangle paradigm with probabilistic imbalances for the present study. The benefit of this paradigm is that it enables one to measure the strength of the objectbased effect in the presence of a more effective, orthogonal strategy (i.e., probabilistic contingencies) and, as such, pushes the system to work at its most efficient setting. Controlling the circumstances under which object-based effects are likely to either manifest or be absent will elucidate the mechanisms that give rise to object-based effects and place further constraints on theories of attentional selection.

\section{EXPERIMENT 1}

In this experiment, we investigated the contribution of exposure duration (i.e., timing) on configural and probability prioritizations. We already know from Shomstein and Yantis (2004) that when SOA is short (i.e., $200 \mathrm{msec}$ ) and the total time that objects are present on the screen is equal to $1,200 \mathrm{msec}$ (i.e., preview time $1,000 \mathrm{msec}$ ), additive effects of both configural and probability prioritization are observed. However, when SOA is longer (i.e., $600 \mathrm{msec}$ ) and the total time that objects are present on the screen is equal to $1,600 \mathrm{msec}$, only probabilities predict RTs. Critically, it is unclear whether the SOA itself or the total exposure duration gives rise to the presence and disappearance of the object-based advantage. In order to examine this timing confound, in this experiment, we introduced another combination of preview time and SOA. Although the short SOA of $200 \mathrm{msec}$ was used again (see Shomstein \& Yantis, 2004, Experiment 2), we reduced the total time that the objects were present on the screen to $400 \mathrm{msec}$ by virtue of shortening the preview time to $200 \mathrm{msec}$. Therefore, in combination with Shomstein and Yantis (2004), we could explore whether the critical predictor of additive effects is (1) the SOA, (2) the total time that objects are present on the screen, (3) the preview time that objects are present on the screen before the onset of the cue, or (4) the combination of preview and SOA.

\section{Method}

Subjects. Two groups of 20 and 16 subjects, respectively, of Carnegie Mellon University undergraduates participated in order to 
fulfill a requirement in a psychology course. All subjects provided informed consent, reported normal or corrected-to-normal visual acuity, and were naive to the purpose of the experiment.

Apparatus and Stimuli. Stimuli were displayed on a 19-in. color monitor, with a viewing distance of approximately $62 \mathrm{~cm}$. A central $0.3^{\circ} \times 0.3^{\circ}$ fixation square and two white rectangle outlines appeared on a black background that was oriented either vertically or horizontally (see Figure 1). Each rectangle subtended $1.2^{\circ} \times 4.2^{\circ}$, with a separation of $1.8^{\circ}$ of visual angle between the rectangles. The cue surrounded one end of one of the rectangles.

One target ( $\mathrm{T}$ or $\mathrm{L}$ ) and three distractors ( $\mathrm{T}-\mathrm{L}$ hybrid) appeared on each trial; each item was centered within the ends of the rectangles. Target letters were constructed with line segments, each subtending $0.6^{\circ}$ of visual angle. Distractor items were created by superimposing the two target letters ( $\mathrm{T}$ and $\mathrm{L}$ ). Target and distractor letters were presented in one of four possible orientations - upright, or rotated $90^{\circ}, 180^{\circ}$, or $270^{\circ}$ (Figure 1). The cue consisted of the appearance of a red outline that perfectly circumscribed one end of one of the rectangles (no gap) for $100 \mathrm{msec}$. Both target and distractor letters were presented in white and subtended $0.6^{\circ} \times 0.6^{\circ}$ of visual angle.

Design and Procedure. A 3 (validity: valid, invalid same-object, invalid different object) $\times 2$ (target-location probability: high, low) $\times 2$ (preview time: 1,000 or $200 \mathrm{msec}$ ) factorial design was used; validity and target location probability were within-subjects factors, and preview time was a between-subjects factor. The design was counterbalanced across subjects for rectangle orientation (vertical or horizontal), absolute location of high- and low-probability lo- cations (both pairs of diagonally opposite positions), and the absolute locations of the possible cue events (upper left and lower right for half of the subjects, lower left and upper right for the other half). Validity was defined by whether the target appeared in the cued location (valid), at the opposite end of the cued object (invalid same-object), or at the end of the uncued rectangle nearest the cue (invalid differentobject). The cue was valid on $50 \%$ of the trials. The target appeared in the invalid high-probability location on $41.7 \%$ of all trials and in the invalid low-probability location on $8.3 \%$ of all trials. Half of the invalid high-probability trials were same-object trials, and the other half were different-object trials; thus, for $50 \%$ of high-probability trials, regardless of which location was cued, the target appeared within the cued object. Similarly, half of the invalid low-probability trials were same-object trials, and the other half were different-object trials.

Each trial began with a display that consisted of a fixation cross and two rectangles that were presented for a preview exposure of either $1,000 \mathrm{msec}$ or $200 \mathrm{msec}$ - a between-subjects manipulation (see Figure 1). The cue was then flashed for $100 \mathrm{msec}$, and after an additional $100 \mathrm{msec}$, the target and three distractors appeared and remained visible until the subject responded. The subjects' task was to identify the target as either T or L (each occurred on a randomly selected half of the trials). Subjects were instructed to ignore the orientation and report only the identity of the target letter, and to respond as quickly as possible while maintaining fixation and about $95 \%$ performance accuracy. The intertrial interval (ITI) was 2,000 msec following each correct response. Each error was fol-
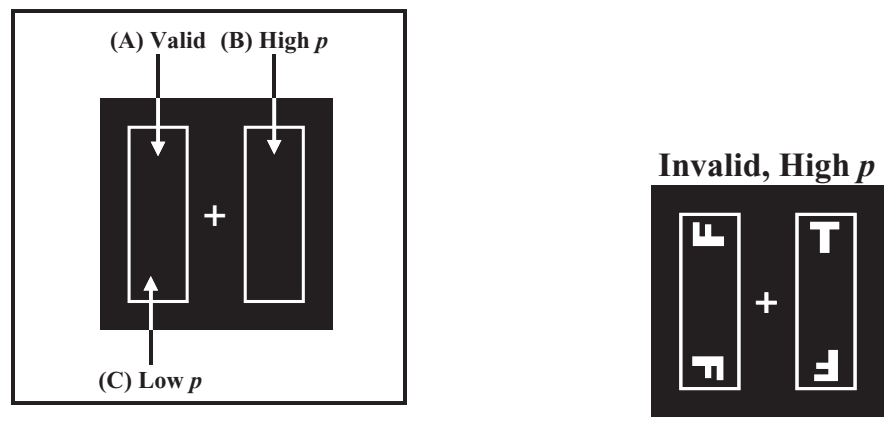

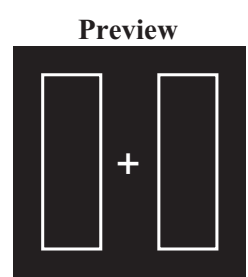

1,000 or $200 \mathrm{msec}$

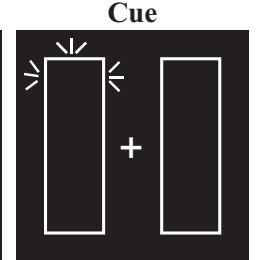

$100 \mathrm{msec}$

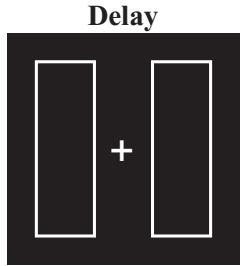

$100 \mathrm{msec}$

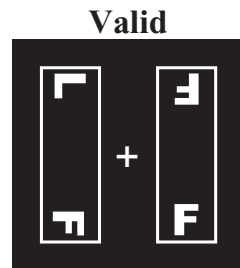

Invalid, Low $p$

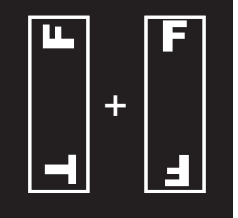

Figure 1. Example display for each of the three conditions of Experiment 1. The task was to perform a $T$ - $-L$ letter discrimination among $T-L$ hybrid distractors. (A) Valid target location: The target appears in the cued end of the rectangle. (B) Invalid high-probability $(p)$ location: upper right. (C) Invalid low-probability location: lower left (this mapping was reversed for half of the subjects). The rectangles were oriented horizontally for half of the subjects; in this case, the high-p/sameobject location becomes the high-p/different-object location, and the low-p/different-object location becomes the low-p/same-object location. 
lowed by visual feedback and an ITI of 4,000 msec. Each subject participated in a 1-h session that consisted of nine blocks of 96 trials, for a total of 864 trials.

\section{Results}

Data from the first two blocks were treated as learning trials and removed from the analysis. Only RTs for correct responses from the remaining blocks were analyzed (overall accuracy 95\%). RTs faster than $200 \mathrm{msec}$ and slower than $2,000 \mathrm{msec}$ were removed from the analysis $(0.2 \%$ and $2.4 \%$, respectively, for preview $1,000 \mathrm{msec}$, and $0.1 \%$ and $2 \%$, respectively, for preview $200 \mathrm{msec}$ ). Data from 3 subjects in the preview $1,000 \mathrm{msec}$ were removed from the analysis due to overall accuracy being lower than $60 \%$. Therefore, analyses were performed on data from 17 subjects in preview $1,000 \mathrm{msec}$, and from 16 subjects in preview $200 \mathrm{msec}$. Preliminary analysis revealed that there were no significant main effects or interactions involving target identity, rectangle orientation, cue locations, or probability locations (all $F_{\mathbf{S}}<1$ ); therefore, the data were collapsed across these variables. Figure 2 summarizes the findings separately for each preview time.

Previews 1,000 and 200 msec. An ANOVA was conducted in order to investigate the interaction between preview time, target-location probability, and same versus different object for the invalidly cued trials for two preview times (i.e., 1,000 vs. $200 \mathrm{msec}$ ). There was a significant main effect of probability $[F(1,31)=72.52, p<$ $.001]$, with faster RTs for high than for low probability locations, and a marginally significant effect of same versus different object $[F(1,31)=3.50, p=.07]$, reflecting the advantage for the same-object location. More importantly, there was no interaction between preview time and target location probability, suggesting that the magnitude of the probability effect ( 75 and $83 \mathrm{msec}$ ) did not change significantly with preview time $(F<1)$. However, there was a marginally significant interaction between preview and same versus different object $[F(1,34)=3.0, p=.08]$ : The magnitude of the same- versus different-object advantage was significant with a preview time of $1,000 \mathrm{msec}$ $(18 \mathrm{msec})$, but not of $200 \mathrm{msec}(1 \mathrm{msec})$.

Preview 1,000 msec. A one-way repeated measures ANOVA revealed a significant main effect of validity $[F(1,16)=40.42, p<.001]$ : RTs for validly cued targets $(M=576 \mathrm{msec})$ were faster than those for invalidly cued targets $(M=648 \mathrm{msec})$, resulting in the overall validity effect of $72 \mathrm{msec}$ (i.e., the difference between the valid and the invalid conditions). The data from the invalidly cued conditions were then subjected to a 2 (invalid high probability and low probability) $\times 2$ (invalid same and different object) repeated measures ANOVA. This analysis revealed a main effect of probability; high-probability targets were identified faster than lowprobability targets $(M=610 \mathrm{msec}$ and $M=685 \mathrm{msec}$, respectively) $[F(1,16)=34.9, p<.001]$, resulting in a probability effect of $75 \mathrm{msec}$. In addition, targets that were presented within the cued object were detected faster than those appearing in the uncued object $(M=$ $639 \mathrm{msec}$ and $M=657 \mathrm{msec}$, respectively) $[F(1,16)=$
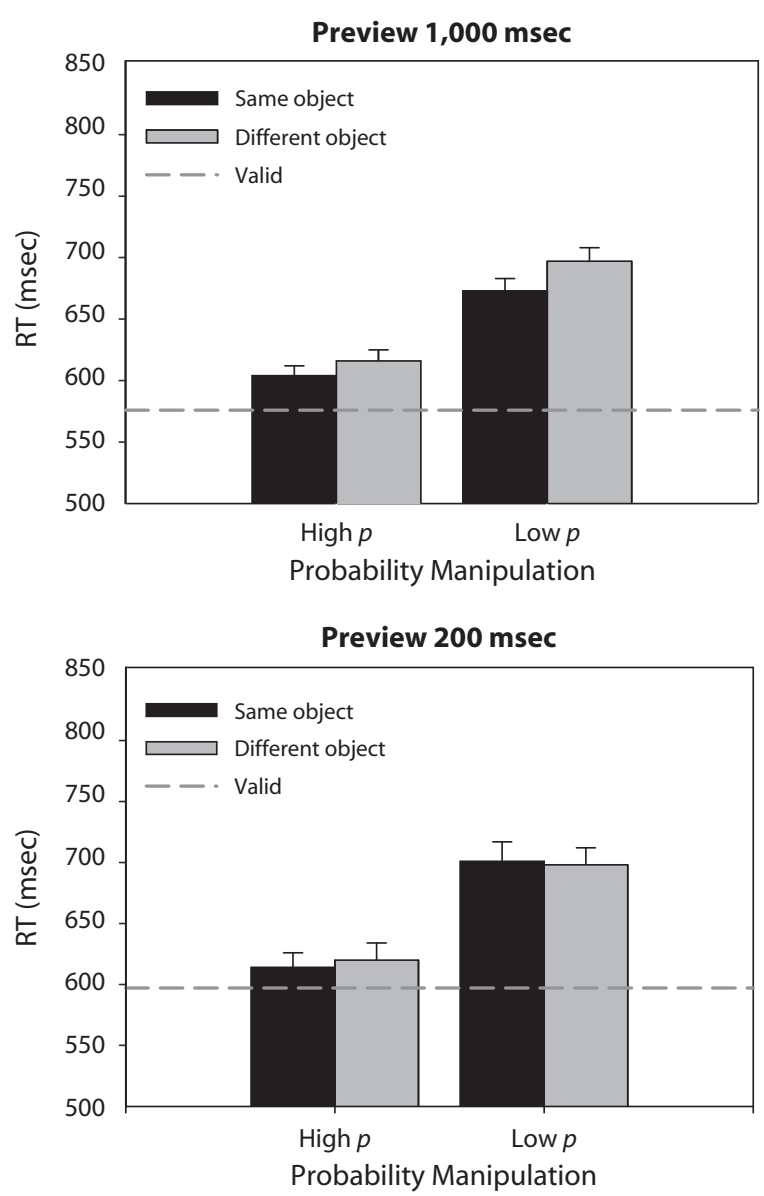

Figure 2. Mean correct reaction times (RTs) for Experiment 1 for preview $1,000 \mathrm{msec}$ and preview $200 \mathrm{msec}$. In each panel, the horizontal dashed line represents the validly cued condition. Error bars represent standard error of the mean.

$6.5, p<.05]$, resulting in an 18 -msec object-based advantage. There was no significant interaction between these factors.

Preview 200 msec. A one-way repeated measures ANOVA revealed a significant main effect of validity $[F(1,15)=29.44, p<.001]$; RTs for validly cued targets $(M=597 \mathrm{msec})$ were faster than those for invalidly cued targets $(M=658 \mathrm{msec})$, resulting in the overall validity effect of $61 \mathrm{msec}$. The data from the invalidly cued conditions were then subjected to a 2 (invalid high probability and low probability) $\times 2$ (invalid same and different object) repeated measures ANOVA. This analysis revealed a main effect of probability; high-probability targets were identified faster than low-probability targets $(M=617 \mathrm{msec}$ and $M=700 \mathrm{msec}$, respectively) $[F(1,15)=37.5, p<$ $.001]$, resulting in a probability effect of $83 \mathrm{msec}$. There was neither a significant main effect of object (1 msecthat is, whether targets appeared within the same or different object as the cue) nor a significant interaction (both $\left.F_{\mathrm{S}}<1\right)$. 


\section{Discussion}

The results of Experiment 1 can be summarized as follows. For preview $1,000 \mathrm{msec}$, we replicated the main finding of Shomstein and Yantis (2004), demonstrating that invalidly cued targets are identified more rapidly when they appear in the high-probability location than when they appear with the low-probability location. The magnitude of the probability effect was $75 \mathrm{msec}$, very similar to the $68 \mathrm{msec}$ reported by Shomstein and Yantis (2004). In addition, invalidly cued targets presented within the cued object were identified more rapidly than those presented in the uncued object, resulting in an object-based advantage of $18 \mathrm{msec}$. Note that the magnitude of the object-based effect (here and in all of the experiments described below) is well within the range of such effects reported in the literature (anywhere from 9 to $30 \mathrm{msec}$ ). Therefore, the primary result of this part of Experiment 1 is a replication of the initial finding that at a preview time of $1,000 \mathrm{msec}$ and a short SOA (200 msec), both probability and objects guide attentional selection.

However, a different pattern of results emerged for the preview 200-msec manipulation. As in preview $1,000 \mathrm{msec}$, subjects identified invalidly cued targets more rapidly when they appeared in the high-probability location than when they appeared in the low-probability location. Note that the magnitude of the probability effects remained relatively unchanged across differences in preview time (75 or $82 \mathrm{msec}$ ), suggesting that the probability manipulation has a consistent and robust effect on performance. In contrast, there was no object-based advantage: RTs were equivalent for the invalidly cued targets appearing within the cued object with those that appeared within the uncued object (i.e., an absence of an object-based effect). Thus, with longer preview time, we observed both effects of local contingencies (i.e., probability based) and of configural (i.e., object-based) factors, whereas when preview time was shortened to $200 \mathrm{msec}$, only probabilities affected performance. According to Shomstein and Yantis's (2004) default configural-setting hypothesis (at a short SOA, both probabilities and configuration guide attentional selection), both types of previews ( $1 \mathrm{sec}$ and $200 \mathrm{msec}$ ) should have led to similar additive effects of objects and probabilities. However, this was not the case. We thus conclude that configural contributions to object-based effects are only observed when the visual system is given enough time to establish a sufficiently robust object representation that can, in turn, guide selection (Shomstein \& Yantis, 2004, Experiment 1A). In addition, if preview time is sufficient, then configuration and probabilities both contribute to selection (preview 1,000-msec condition here, and Experiment 1A in Shomstein and Yantis, 2004); however, if enough time is given to the visual system to suppress the established configural representation (i.e., long SOA), then this configural prioritization is abandoned in favor of probabilities (Shomstein \& Yantis, 2004).

\section{EXPERIMENT 2}

In Experiment 1, we demonstrated that only the location probabilities and not the object structure guide at- tention when preview time is decreased from $1 \mathrm{sec}$ to $200 \mathrm{msec}$. A possible explanation for why apparently ubiquitous object-based effects are absent in a shorter preview time condition might simply be that $200 \mathrm{msec}$ is not long enough to establish an object representation. If this were so, the conclusion would be that probabilistic guidance of attentional selection is immune to object structure because of insufficient time to establish the object structure. In order to test this hypothesis, we removed the probability manipulation from the design. If the object-based effect is obtained under these conditions (i.e., short preview time and no probabilistic imbalance), then we would conclude that $200 \mathrm{msec}$ is indeed sufficient to obtain the object advantage, but that in the presence of a probability manipulation, its contribution is abandoned in lieu of a more efficient search strategy.

\section{Method}

Subjects. Nineteen new Carnegie Mellon University undergraduates participated in this experiment in order to fulfill a requirement in a psychology course. All reported normal or corrected-to-normal visual acuity and were naive to the purpose of the experiment.

Apparatus and Stimuli. The stimuli were the same as those in Experiment 1

Design and Procedure. The design was same as that in Experiment 1 , with the only difference being that the probability manipulation was removed; therefore, the appearance of targets was equally likely $(25 \%$ each) in invalidly cued locations. This is the standard object-based attention paradigm, but with preview time fixed at $200 \mathrm{msec}$. All other elements of the design were kept the same as those in Experiment 1.

\section{Results and Discussion}

Data from the first two blocks were removed in order to ensure consistency with the analysis reported for Experiment 1 . RTs that were faster than $200 \mathrm{msec}$ and slower than $2,000 \mathrm{msec}$ were removed from the analysis $(0.1 \%$ and $1.4 \%$, respectively). A preliminary analysis revealed neither significant main effects nor interactions involving target identity, rectangle orientation, or cue locations (all $F$ s $<1$ ); therefore, the data were collapsed across these variables (see Figure 3).

A one-way repeated measures ANOVA revealed a significant main effect of validity; RTs for validly cued targets $(M=589 \mathrm{msec})$ were detected faster than those for invalidly cued targets $(631 \mathrm{msec})[F(1,18)=39.86, p<.001]$, resulting in the overall validity effect of $42 \mathrm{msec}$ (see Table 1). Furthermore, an ANOVA revealed a significant effect of object; invalid targets presented within the cued object $(M=621 \mathrm{msec})$ were detected faster than those presented in the uncued object $(M=641 \mathrm{msec})[F(1,18)=$ $5.6, p<.05]$ - an object-based effect of $20 \mathrm{msec}$.

Importantly, the results from this experiment revealed that if preview time is $200 \mathrm{msec}$ and the probabilistic imbalance is removed from the two-rectangle display so that invalidly cued targets are just as likely to appear in the invalidly cued object as in the validly cued object, objects can indeed guide attentional selection, and object-based attentional modulations are observed. Thus, the failure to observe the object-based benefit in Experiment 1 cannot be attributed to the inadequate derivation of an object-based representation; rather, it may be explained by the overpowering ef- 


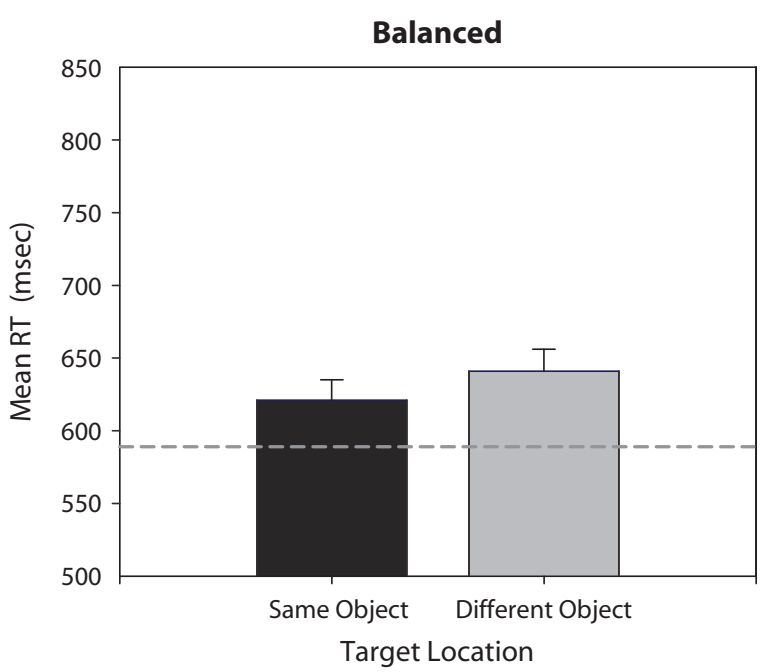

Figure 3. Mean correct reaction times (RTs) for Experiment 2 in which targets appeared in each of the invalidly cued locations equiprobably. The horizontal dashed line represents the validly cued condition.

fect of the probability manipulation, which favors the high probability location independent of its object status.

\section{EXPERIMENT 3}

Several conclusions regarding the effect of time on the object-based attentional modulation can be made at this juncture. When preview time is long (i.e., 1,000 msec) and the probabilistic imbalance is imposed onto the tworectangle display, probabilities as well as configuration (i.e., objects) guide attentional selection (see Table 1). Moreover, the effects of probability and objects are additive, as is evidenced by the absence of interactive effects. However, when the preview time is decreased to $200 \mathrm{msec}$, objects fail to exert their influence on attentional guidance, and probability is the only factor that predicts subjects' RTs. In addition, the failure to observe object-based effects at this short preview cannot be explained by the fact that object representations cannot be established in

Table 1

Summary

\begin{tabular}{lrrrr}
\hline \multicolumn{1}{c}{ Description } & $\begin{array}{r}\text { Preview } \\
(\mathrm{msec})\end{array}$ & Valid & Prob. & OB \\
\hline Object outlines & 1,000 & 72 & 75 & 18 \\
$\quad$ (Exp. 1) & 200 & 61 & 82 & 1 \\
Balanced (probabilities removed) & & & & \\
$\quad$ (Exp. 2) & 200 & 42 & $\mathrm{NA}$ & 20 \\
Objects filled in with red & 1,000 & 84 & 104 & 21 \\
$\quad$ (Exp. 3) & 200 & 65 & 98 & 0 \\
Objects are red and blue & & & & \\
$\quad$ (Exp. 4) & 200 & 67 & 70 & 26 \\
Object outlines (detection) & 1,000 & -1 & 11 & 12 \\
$\quad$ (Exp. 5) & 200 & 8 & 13 & 13 \\
\hline
\end{tabular}

Note-Prob., probability; OB, object-based.
$200 \mathrm{msec}$ : The results of Experiment 2 indicate that when the probabilistic imbalance is removed, object-based effects do emerge at this exposure duration.

One possible explanation for the dominance of the probabilistic strategy in the experiments reported previously is that the "objects" used here are relatively weak in "objecthood," and as such, they cannot exert any influence in the presence of the overpowering probability manipulation. The two-rectangle displays consisted merely of outlines of two rectangles - a white boundary on a black background that groups the area inside it by a common region (Palmer \& Rock, 1994). One way to enhance the "objecthood" of the rectangles and make them easier to segment from the background is to enhance their surface properties by filling them in with a salient color (e.g., red). This manipulation creates a percept of two red rectangles positioned on top of the uniform black background-a manipulation that aids perceptual organization.

The prediction is as follows: If the weakness of the object-based representation prevented the configuration from modulating attentional orienting under probability manipulations, then by making objects more salient, we might still be able to recover object-based effects even under brief preview.

\section{Method}

Subjects. Fifteen (for preview 1,000) and 16 (for preview 200) new Carnegie Mellon University undergraduates participated in this experiment in order to fulfill a requirement in a psychology course. All reported normal or corrected-to-normal visual acuity and were naive to the purpose of the experiment.

Apparatus and Stimuli. The stimuli were same as those in Experiment 1 , with previews of 1,000 and $200 \mathrm{msec}$, and an SOA of $200 \mathrm{msec}$.

Design and Procedure. The design was the same as that in Experiment 1 , with the only difference being that the two objects were now colored red (and the white outline around the rectangles was removed). Therefore, the percept was of two red rectangles presented on the screen either vertically or horizontally.

\section{Results and Discussion}

Data from the first two blocks were treated as learning trials and were removed from the analysis. Only RTs for correct responses were analyzed. RTs faster than $200 \mathrm{msec}$ and slower than 2,000 msec were removed from the analysis $(0.17 \%$ and $3 \%$, respectively). Preliminary analysis revealed that there were no significant main effects or interactions involving target identity, rectangle orientation, cue locations, or probability locations (all $F_{\mathrm{S}}<1$ ); therefore, the data were collapsed across these variables (see Figure 4).

Previews 1,000 and 200 msec. An ANOVA was conducted in order to investigate the interaction between preview time, target-location probability, and same versus different object for the invalidly cued trials for two preview times (i.e., 1,000 vs. $200 \mathrm{msec}$ ). There was a significant main effect of probability $[F(1,29)=68.58, p<$ $.001]$, with faster RTs for high than for low probability locations, and of same- versus different-object $[F(1,29)=$ $6.28, p=.02]$, reflecting the advantage for the sameobject location. More importantly, there was no interaction between preview time and target-location probability, 

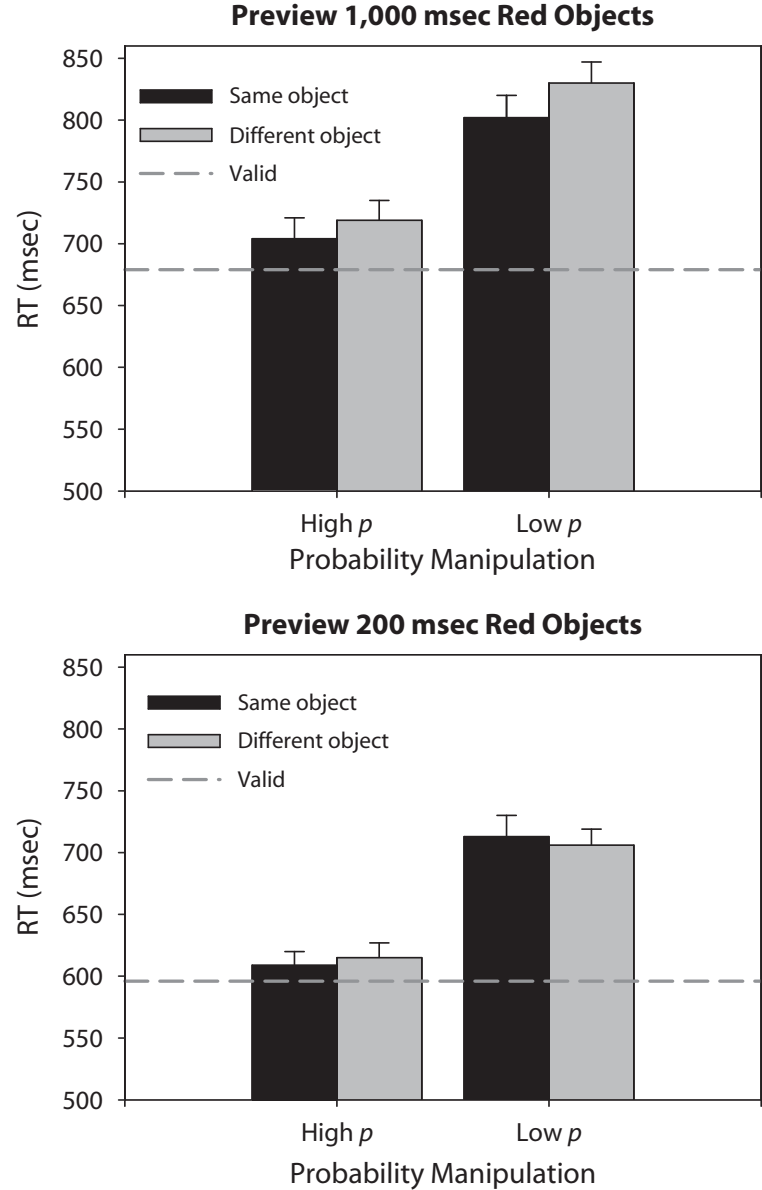

Figure 4. Mean correct reaction times (RTs) from Experiment 3 for preview 1,000 msec and preview $200 \mathrm{msec}$. In each panel, the horizontal dashed line represents the validly cued condition. Error bars represent standard error of the mean.

suggesting that the magnitude of the probability effect ( 75 and $83 \mathrm{msec})$ did not change significantly with preview time $(F<1)$. However, there was a significant $(p<.01)$ interaction between preview and same versus different object $[F(1,29)=6.67, p=.02]$. The magnitude of the same- versus different-object advantage was significant with a preview time of $1,000 \mathrm{msec}(21 \mathrm{msec})$, but not of $200 \mathrm{msec}(0 \mathrm{msec})$.

Preview 1,000 msec. A one-way repeated measures ANOVA revealed a significant main effect of validity $[F(1,14)=25.58, p<.001]$; RTs for validly cued targets $(M=679 \mathrm{msec})$ were faster than those for invalidly cued targets $(M=764 \mathrm{msec})$, resulting in the overall validity effect of $84 \mathrm{msec}$ (i.e., the difference between the valid and the invalid conditions). The data from the invalidly cued conditions were then subjected to a 2 (invalid high probability and low probability) $\times 2$ (invalid same and different object) repeated measures ANOVA. This analysis revealed a main effect of probability; high-probability targets were identified faster than low-probability targets ( $M=712 \mathrm{msec}$ and $M=816 \mathrm{msec}$, respectively)
$[F(1,14)=28.18, p<.001]$, resulting in a probability effect of $104 \mathrm{msec}$. In addition, targets presented within the cued object were detected faster than those appearing in the uncued object ( $M=753 \mathrm{msec}$ and $M=774 \mathrm{msec}$, respectively) $[F(1,14)=22.95, p<.001]$, resulting in a 21 -msec object-based advantage. There was no significant interaction between these factors.

Preview 200 msec. A one-way repeated measures ANOVA revealed a significant main effect of validity $[F(1,15)=19.16, p=.001]$; RTs for validly cued targets $(M=596 \mathrm{msec})$ were faster than those for invalidly cued targets $(M=661 \mathrm{msec})$, resulting in an overall validity effect of $65 \mathrm{msec}$ (see Table 1). The data from the invalidly cued conditions were then subjected to a 2 (invalid high probability and low probability) $\times 2$ (invalid same and different object) repeated measures ANOVA. This analysis revealed a main effect of probability; high-probability targets were identified faster than lowprobability targets $(M=612 \mathrm{msec}$ and $M=710 \mathrm{msec}$, respectively) $[F(1,15)=43.70, p<.001]$, resulting in a probability effect of $98 \mathrm{msec}$. No other main effect or interaction was significant (both $F_{\mathrm{s}}<1$ ).

Results from this experiment indicate that despite making the objects more salient by coloring the rectangles with a uniform color, a preview time of $200 \mathrm{msec}$ still failed to elicit object-based attentional effects. As in Experiment 1, at preview $200 \mathrm{msec}$, RTs were solely predicted by the probabilistic imbalance that was imposed onto the tworectangle display, and the presence of objects in the display was irrelevant. A question then remains: Is there a salience manipulation that might bring back object-based effects under such short preview time?

\section{EXPERIMENT 4}

Results from the first three experiments point to two variables that play a crucial role in determining the mechanisms that guide attentional selection. First, we demonstrated that configuration exerts its influence on attentional guidance when invalid-same and invalid-different object targets are equiprobable. However, once a probabilistic imbalance is introduced to the scene, object-based guidance seems to be abandoned in lieu of a more predictable attentional guidance (if, of course, enough time is given for the object-based representation to evolve, as is the case with 1,000-msec preview time).

The next manipulation involved one further attempt to increase the strength of the representation of objects by making the two objects distinct from one another. The logic was to attempt to bring out the configural guidance even in the presence of probabilistic imbalances by introducing two dissimilar objects. Therefore, in this experiment, we colored one of the rectangles red and the other blue. In this manipulation, when the two rectangles are of different color, the cue not only cues the location and the object, but also the color, thus providing greater differences between the attended and unattended objects.

The predictions were as follows. Given that the cue not only cues the location and the object but also the color, there should be a cost associated with detecting a target in 
the invalidly cued object (i.e., associated with switching to a different color). Or, there could be a facilitation of an attentional shift within the same color; therefore, we should observe both the probability effect and the object-based effect. Note that when the preview is $200 \mathrm{msec}$ and the SOA is $200 \mathrm{msec}$, this experiment matches the parameters of Experiments 1 and 3. Recall that in neither of these cases (when two rectangles were the same color, either both black or both red) did we observe object-based modulations. Therefore, the question for this experiment, given that the two objects are different, is whether there will be a reduction in the cost for switching to the other object (i.e., will only probabilities guide attention)?

\section{Method}

Subjects. Fifteen new Carnegie Mellon University undergraduates participated in this experiment in order to fulfill a requirement in a psychology course. All reported normal or corrected-to-normal visual acuity and were naive to the purpose of the experiment.

Apparatus and Stimuli. The stimuli were same as those in Experiment 1 (preview $200 \mathrm{msec}$ ), with the only difference being that one of the objects was filled in with the color red and the other with the color blue (again, the white outline around the rectangles was removed). Half of the time the blue object was on the left (or top), and the other half of the time it was on the right (or bottom).

Design and Procedure. A 3 (validity: valid, invalid same-object, invalid different-object) $\times 2$ (target-location probability: high, low) factorial design was used, with validity and target-location probability as within-subjects factors. Aside from these changes, the design and procedure were the same as those in Experiments 1 and 3.

\section{Results and Discussion}

Data from the first two blocks were treated as learning trials and removed from the analysis. Only RTs for correct responses were analyzed. RTs faster than $200 \mathrm{msec}$ and slower than $2,000 \mathrm{msec}$ were removed from the analysis $(0.19 \%$ and $2.5 \%$, respectively). A preliminary analysis revealed that there were no significant main effects or interactions involving target identity, rectangle orientation, cue locations, color position, or probability locations (all $F_{\mathrm{S}}<1$ ); therefore, the data were collapsed across these variables. Figure 5 summarizes the findings (see also Table 1).

A one-way repeated measures ANOVA revealed a significant main effect of validity $[F(1,14)=56.89$, $p<.001]$; RTs for validly cued targets $(M=568 \mathrm{msec})$ were faster than those for invalidly cued targets $(M=$ $635 \mathrm{msec}$ ), resulting in the overall validity effect of $67 \mathrm{msec}$. The data from the invalidly cued conditions were then subjected to a 2 (invalid high probability and low probability) $\times 2$ (invalid same and different object) repeated measures ANOVA. This analysis revealed a main effect of probability; high-probability targets were identified faster than low-probability targets $(M=600 \mathrm{msec}$ and $M=670 \mathrm{msec}$, respectively) $[F(1,14)=33.20, p<$ $.001]$, resulting in a probability effect of $70 \mathrm{msec}$. The ANOVA also revealed a significant effect of object; targets presented within the cued object $(M=622 \mathrm{msec})$ were detected faster than those presented in the uncued object $(M=648 \mathrm{msec})[F(1,14)=11.36, p<.01]$-an object-based effect of $26 \mathrm{msec}$. Furthermore, there was a significant interaction between probability and same ver- sus different object $[F(1,14)=5.15, p=.04]$; the magnitude of the object-based effect was largest at the lowprobability location (38-msec effect) as compared with the high-probability location (13-msec effect).

In addition, we conducted an ANOVA with probability and object as within-subjects factors and with object-type (i.e., strength of representation) from $200-\mathrm{msec}$ preview only (i.e., outlines from Experiment 1, red filled-in objects from Experiment 3, and red and blue objects from Experiment 4). We observed a main effect of probability $[F(1,44)=113, p<.001]$ and of object $[F(1,44)=4.33$, $p<.05]$. There was also a significant interaction of object type with same-different object $[F(2,44)=4.01, p<$ .03]. No other main effects or interactions were significant $(F<1)$. Note that probability manipulation did not interact with object type, suggesting that the magnitude of the probability manipulation is pretty much constant across all of the experiments.

Results of this experiment in which the strength of the object representation was manipulated by coloring the two objects with two distinct colors (i.e., red and blue) revealed that both configuration and probabilities were guiding attentional selection. In addition, there was a significant interaction between the objects and probabilities; the magnitude of the object-based attentional modulation was greater at the low-probability location than at the high-probability location. Therefore, we conclude that short exposure durations (i.e., preview time of $200 \mathrm{msec}$ ) can give rise to configural and probabilistic guidance only if object representations are sufficiently robust (also evidenced by the between-experiment ANOVA), which was achieved here by increasing the dissimilarity between the attended and unattended object.

\section{EXPERIMENT 5}

In the experiments described up to this point, we demonstrated that probabilistic and configural prioritizations are employed in attentional guidance that requires finegrained discrimination of target stimuli embedded among

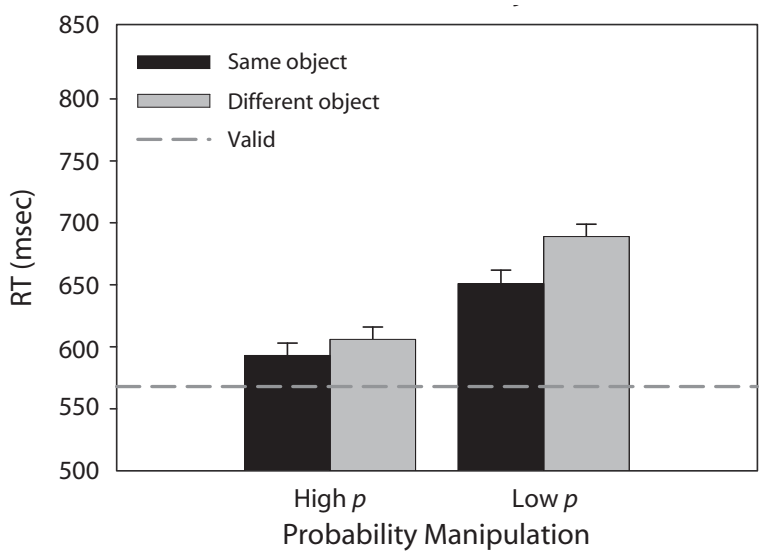

Figure 5. Mean correct reaction times (RTs) from Experiment 4. RT to validly cued targets is represented by the horizontal dashed line. 
similar distractors. In this final experiment, we examined whether the probabilistic and configural prioritizations exert their influences in a task that does not require target identification and that also does not rely on visual search - that is, a target-detection task. One could argue that strategies that are brought to bear in a task involving time-consuming search may be quite different than those employed in a simple detection task. As in Experiments 1 and 3, we manipulated preview time between 1,000 and $200 \mathrm{msec}$ in order to investigate whether, and if, these two strategic components are used to establish a priority map in a simple detection task.

\section{Method}

Subjects. Thirty new Carnegie Mellon University undergraduates participated in this experiment in order to fulfill a requirement in a psychology course. All reported normal or corrected-to-normal visual acuity and were naive as to the purpose of the experiment.

Apparatus and Stimuli. The stimuli were same as those in Experiment 1 (rectangles with white outlines, with a preview of either 1,000 or $200 \mathrm{msec}$ ). In contrast with the previous target discrimination experiment, in the present experiment, subjects were required to detect the presence of the white square that was flashed in one of three possible target locations. The target square subtended $0.6^{\circ} \times$ $0.6^{\circ}$ of visual angle and remained on the screen until the response. Ten percent of the trials were catch trials; the white square was not presented and subjects had to withhold their response.

Design and Procedure. A 3 (validity: valid, invalid same-object, invalid different-object) $\times 2$ (target location probability: high, low) factorial design was used with validity and target location probability as within-subjects factors. Aside from these changes, the design and procedure were the same as those in Experiments 1, 3, and 4.

\section{Results and Discussion}

Data from the first two blocks were treated as learning trials and were removed from the analysis. Only RTs for correct responses were analyzed. RTs that were faster than $150 \mathrm{msec}$ and slower than $1,000 \mathrm{msec}$ were removed from the analysis ( $3 \%$ and $0.98 \%$, respectively). Different thresholds were used in this experiment in order to accommodate the rapid RTs that accompany detection tasks. A preliminary analysis revealed that there were no significant main effects or interactions involving rectangle orientation, cue locations, or probability locations (all $F \mathrm{~s}<1$ ); therefore, the data were collapsed across these variables. Figure 6 summarizes the findings (see also Table 1).

Previews 1,000 and 200 msec. An ANOVA was conducted in order to investigate the interaction between preview time, target location probability, and same versus different object for the invalidly cued trials for two preview times (i.e., 1,000 vs. $200 \mathrm{msec}$ ). There was a significant main effect of probability $[F(1,28)=23.46, p<$ $.001]$, with faster RTs for high- $(M=319 \mathrm{msec})$ than for low-probability $(M=330 \mathrm{msec})$ locations, and of same $(M=318 \mathrm{msec})$ versus different $(M=331 \mathrm{msec})$ object $[F(1,29)=6.28, p=.02]$, reflecting the advantage for the same-object location. More importantly, there were no interactions between preview time and target-location probability or same versus different object, suggesting that the magnitude of both the probability effect and the object effect did not change significantly with preview time $(F<1)$.
Preview 1,000 msec. A one-way repeated measures ANOVA revealed a significant main effect of validity $[F(1,14)=5.23, p<.05]$; RTs for validly cued targets $(M=316 \mathrm{msec})$ were faster than those for invalidly cued targets $(M=324 \mathrm{msec})$, resulting in the overall validity effect of $8 \mathrm{msec}$. The data from the invalidly cued conditions were then subjected to a 2 (invalid high probability and low probability) $\times 2$ (invalid same and different object) repeated measures ANOVA. This analysis revealed a main effect of probability; high-probability targets were identified faster than low-probability targets $(M=$ $319 \mathrm{msec}$ and $M=330 \mathrm{msec}$, respectively) $[F(1,14)=$ $8.30, p<.05]$, resulting in a probability effect of $11 \mathrm{msec}$. In addition, targets presented within the cued object were detected faster than those appearing in the uncued object ( $M=318 \mathrm{msec}$ and $M=330 \mathrm{msec}$, respectively) $[F(1,14)=26.51, p<.001]$, resulting in a $12-\mathrm{msec}$ object-based advantage. There was no significant interaction between these factors.

Preview 200 msec. A one-way repeated measures ANOVA did not reveal a significant main effect of valid-
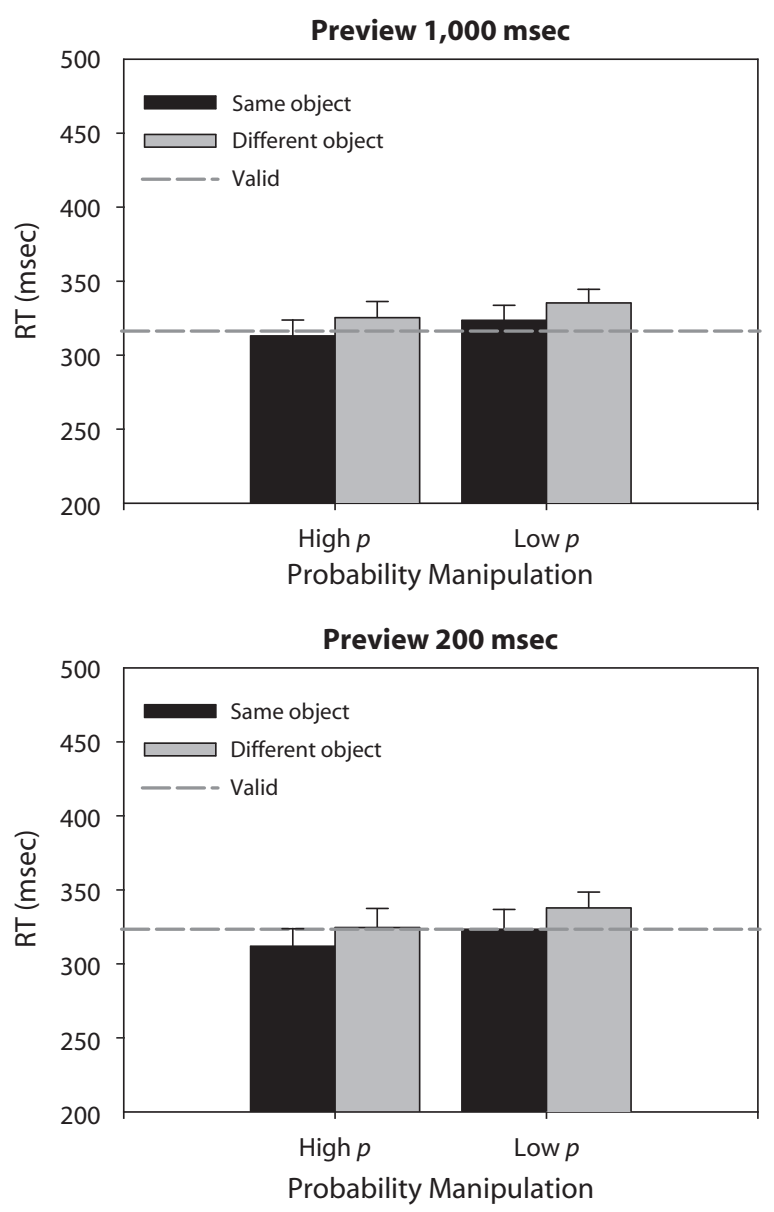

Figure 6. Mean correct reaction times (RTs) from the detection Experiment 5. RT to validly cued targets is represented by the horizontal dashed line. 
ity $(F<1)$, suggesting that the cue was not effective in enhancing the subject's attention.

The data from the invalidly cued conditions were then subjected to a 2 (invalid high probability and low probability) $\times 2$ (invalid same and different object) repeated measures ANOVA. This analysis revealed a main effect of probability; high-probability targets were identified faster than low-probability targets $(M=318 \mathrm{msec}$ and $M=$ $331 \mathrm{msec}$, respectively) $[F(1,14)=16.82, p<.001]$, resulting in a probability effect of $13 \mathrm{msec}$. No other main effect or interaction was significant (both $F \mathbf{s}<1$ ). In addition, targets presented within the cued object were detected faster than those appearing in the uncued object ( $M=318 \mathrm{msec}$ and $M=331 \mathrm{msec}$, respectively) $[F(1,14)=35.73, p<.001]$, resulting in a $13-$ msec objectbased advantage. There was no significant interaction between these factors.

The results of this experiment suggest that both prioritizations - configural and probabilistic - are brought to bear even in a simple detection task, just as in a target identification task. Several differences with a target identification task were nonetheless observed. First, the strength of the probabilistic advantage (i.e., the benefit for the high-probability location) was greatly reduced (from a 70- to 100-msec advantage to an 11-msec advantage) in comparison with the probability advantage in a target identification task. This reduction in probability guidance is not surprising, given that overall RTs were roughly twice as fast in the target-detection task than in the target-discrimination task. The second - and perhaps the most interesting - difference between the two tasks is that configural prioritization exerts its influence even when the preview time is $200 \mathrm{msec}$. It appears as if a 200msec preview time is sufficient to establish an object-like configuration that can be used to guide detection, whereas the same object-like configuration is not sufficiently powerful to exert its effect in the more difficult targetdiscrimination search task. This interaction between the strength of object representation and its ability to guide attentional selection in tasks of various difficulty warrants a more careful examination in future studies.

\section{GENERAL DISCUSSION}

In the present study, we investigated factors that affect attentional selection by examining the contribution of two different hypothesized attentional prioritization processes to object-based selection. ${ }^{1}$ The first prioritization account was based purely on configuration (i.e., objects) that was present in the display. This configuration-based mechanism (Shomstein \& Yantis, 2004) predicts that targets presented in the invalid same-object location are detected faster than those presented within the invalid different-object locations, because higher attentional priority was assigned to locations that were bound by a common region (i.e., same object location) rather than to locations elsewhere within the display. The second prioritization process (Avrahami, 1999; Moore et al., 1988; Shomstein \& Yantis, 2002, 2004) operates on probabilistic imbalances and predicts that targets presented in the high-probability location will be de- tected faster than targets presented in the low-probability location, regardless of whether these targets appear within the cued object or in a different object.

In a set of five experiments, we demonstrated that attentional guidance is a dynamic process in which no single mechanism constitutes a frank default setting. Rather, attentional guidance is computed on the basis of relative strengths of object representations, as well as the local contingencies of the environment at hand, and task difficulty. When objects are saliently individuated (i.e., two objects are of different colors), both configural and probabilistic prioritizations guide attentional selection, even under brief preview time. However, we also demonstrated several instances in which attentional prioritization (based on local contingencies of the scene) alone appears to guide attentional performance (e.g., Experiments 1 and 3: the 200-msec preview time manipulation).

Recently, Müller and Kleinschmidt (2003) offered neuroimaging evidence for the close interaction between configural and probabilistic attentional prioritizations. ${ }^{2}$ The authors adopted a two-rectangle method while measuring activity in early visual cortex with fMRI. The authors recorded activity in the early sensory visual regions that corresponded to the four possible target locations (i.e., upper left, upper right, lower right, and lower left). If the upcoming target event appeared at the expected location (i.e., the cued location - upper left), then there was little activity increase at the other locations (lower right and left, and upper right). However, if the target did not appear at the expected location, then a substantial signal increase was observed in representations of locations that belonged to the same object, but not of those belonging to the other object. This type of increased activity within the same-object location would be predicted by either the configural prioritization strategy or the probabilistic prioritization strategy. As in the Egly et al. (1994) two-rectangle study, in the study by Müller and Kleinschmidt, the probability of target occurrences was mostly stacked within the cued object.

In this article, we identified the strength of object representation as an additional crucial factor that determines which attentional prioritization will guide selection. In our Experiments 1 and 2, in conjunction with those of Shomstein and Yantis (2004), we demonstrated that exposure duration (in particular, preview time) influences object representations so that both configural and probabilitybased strategies guide selection when preview time is long (i.e., objects exposed on the screen for $1 \mathrm{sec}$ before the onset of the cue), as well as when SOA is long (i.e., $600 \mathrm{msec}$ ). However, what is important is that the critical variable that is instrumental to abandoning the configural guidance is not simply the reduced preview time-because objects guide attention when probabilistic imbalances are removed in Experiment 2 - but that preview-time manipulation along with probabilistic imbalances reduces the influence that objects exert on attentional guidance.

The next set of manipulations controlled object-based attentional guidance by simply varying the strength of object representations. In Experiment 3, we showed that simply enhancing object representations by making objects more salient (i.e., coloring the objects red) did not invoke object- 
based guidance unless preview time was increased again to $1,000 \mathrm{msec}$. However, object-based effects could be made to exert their influence on both attentional guidance with probabilistic imbalances superimposed and the short preview time by simply reducing the similarity between the two objects. In Experiment 4, when the two objects differed in color (i.e., one of the objects was blue, and the other was red), both probability and objects guided attentional selection.

Note, however, that the two-color manipulation that was employed in Experiment 4 might have given rise to object-based effects via an alternative mechanism. The sensory cue that was presented quickly after the onset of the objects might have cued a feature (i.e., red or blue) rather than an object per se, thus benefiting same-feature location rather than same-object location. Whatever the case may be - cued feature or cued object - the results of Experiment 4 demonstrated that even with the probabilistic imbalance imposed onto the visual scene, it is possible to elicit object-based or feature-based benefit for items (object or features) that are only previewed shortly (i.e., $200 \mathrm{msec}$ ). It might be of interest for future research to examine in greater detail what pictorial cues (other than coloring the two objects with different colors) might increase the object representation sufficiently in order to give rise to both object-based and probability-based effects.

Finally, we demonstrated that the extent to which the probability and configural prioritization influence attentional guidance depends, among other things, on task difficulty. Whereas probability modulated attention to a greater extent in discrimination tasks, its benefit in the detection task was much reduced $(90 \mathrm{msec}$ to $10 \mathrm{msec}$, respectively). In addition, the magnitude of the configural contribution remained equally effective regardless of task difficulty, and it did not seem to be affected by the time available to establish an object-based representation. This last finding, however, might only apply to preview times greater than $200 \mathrm{msec}$ (examined here), and presumably, if the preview time were to be decreased even further, at some point, configural prioritization would fail to exert its influence.

In summary, on the basis of the present experiments, as well as several others that investigated the mechanism of object-based attentional selection, we suggest that objectbased effects result from a combination of configural and probabilistic prioritizations. In order for an object-based benefit to develop, the visual system needs to operate on an object representation that - as evident from the results of these studies - needs time to develop (i.e., object-based effects are not present at the 200-msec preview time unless it is a detection task, and are present when 1,000 msec are given for a preview). If ample time is provided for developing a strong enough object representation, then both objects and probabilities will guide attentional selection. However, the contribution of configural prioritization can be suppressed (i.e., no object-based effects) only if (1) there is ample time for object-based representation to develop (Experiments 1 and 3), and (2) there is enough time available between the cue and the oncoming target (Experiment 2 in Shomstein and Yantis, 2004).
As research investigations of object-based attentional selection move beyond simply showing that object-based effects do exist, the field is moving closer to understanding the mechanism of attentional guidance. Recent neuroimaging studies suggest that attentional guidance is a dynamic process that takes into account the local contingencies of the scene as well as the object configurations (Müller \& Kleinschmidt, 2003; O’Craven et al., 1999; Serences, Schwarzbach, Courtney, Golay, \& Yantis, 2004; Shomstein \& Behrmann, 2006). Experiments reported in this article examine just how dynamic this attentional guidance is. We suggest that the frequently observed object-based attentional modulation effects are more complex than generally believed and, further, that attentional selection is not necessarily guided by both space and objects; rather, it is a dynamic process of computing local contingencies and strengths of representation of objects that are present in the environment.

\section{AUTHOR NOTE}

This research was supported in part by National Institute of Neurological Disorders and Stroke Grant NS07391-09 to S.S. and National Institute of Mental Health Grant MH54246 to M.B. We thank Tom Carr, Tom Sanocki, and an anonymous reviewer for helpful comments on earlier versions of this work. Correspondence should be addressed to S. Shomstein, Department of Psychology, George Washington University, Washington, DC 20052 (e-mail: shom@gwu.edu).

\section{REFERENCES}

Avrahami, J. (1999). Objects of attention, objects of perception. Perception \& Psychophysics, 61, 1604-1612.

Behrmann, M., Zemel, R. S., \& Mozer, M. C. (1998). Object-based attention and occlusion: Evidence from normal participants and a computational model. Journal of Experimental Psychology: Human Perception \& Performance, 24, 1011-1036.

CAVE, K. R., \& Wolfe, J. M. (1990). Modeling the role of parallel processing in visual search. Cognitive Psychology, 22, 225-271.

Cepeda, N. J., \& Kramer, A. F. (1999). Strategic effects on objectbased attentional selection. Acta Psychologica, 103, 1-19.

Chun, M. M., \& JiAng, Y. (1998). Contextual cueing: Implicit learning and memory of visual context guides spatial attention. Cognitive Psychology, 36, 28-71.

Corbetta, M., Miezin, F. M., Dobmeyer, S., Shulman, G. L., \& PeTERSEN, S. E. (1990). Attentional modulation of neural processing of shape, color, and velocity in humans. Science, 248, 1556-1559.

Desimone, R., \& Duncan, J. (1995). Neural mechanisms of selective visual attention. Annual Review of Neuroscience, 18, 193-222.

Downing, C. J., \& Pinker, S. (1985). The spatial structure of visual attention. In M. I. Posner \& O. S. M. Marin (Eds.), Attention and performance XI (pp. 171-188). Hillsdale, NJ: Erlbaum.

Duncan, J. (1984). Selective attention and the organization of visual information. Journal of Experimental Psychology: General, 113, 501-517.

Egly, R., Driver, J., \& Rafal, R. D. (1994). Shifting visual attention between objects and locations: Evidence from normal and parietal lesion subjects. Journal of Experimental Psychology: General, 123, 161-177.

Eriksen, C. W., \& St. James, J. D. (1986). Visual attention within and around the field of focal attention: A zoom lens model. Perception \& Psychophysics, 40, 225-240.

Geng, J. J., \& Behrmann, M. (2002). Probability cuing of target location facilitates visual search implicitly in normal participants and patients with hemispatial neglect. Psychological Science, 13, 520-525

JiAng, Y., \& Chun, M. M. (2001). Selective attention modulates implicit learning. Quarterly Journal of Experimental Psychology, 54A, 1105-1124. 
Kahneman, D., \& Henik, A. (1981). Perceptual organization and attention. In M. Kubovy \& J. R. Pomerantz (Eds.), Perceptual organization (pp. 181-211). Hillsdale, NJ: Erlbaum.

Kastner, S., De Weerd, P., Desimone, R., \& Ungerleider, L. G. (1998). Mechanisms of directed attention in the human extrastriate cortex as revealed by functional MRI. Science, 282, 108-111.

Marrara, M. T., \& Moore, C. M. (2003). Object-based selection in the two-rectangles method is not an artifact of the three-sided directional cue. Perception \& Psychophysics, 65, 1103-1109.

Miller, J. (1988). Components of the location probability effect in visual search tasks. Journal of Experimental Psychology: Human Perception \& Performance, 14, 453-471.

Moore, C. M., \& Egeth, H. (1998). How does feature-based attention affect visual processing? Journal of Experimental Psychology: Human Perception \& Performance, 24, 1296-1310.

Moore, C. M., Yantis, S., \& Vaughan, B. (1988). Object-based visual selection: Evidence from perceptual completion. Psychological Science, 9, 104-110.

Müller, N. G., Bartelt, O. A., Donner, T. H., Villringer, A., \& BRANDT, S. A. (2003). A physiological correlate of the "zoom lens" of visual attention. Journal of Neuroscience, 23, 3561-3565.

Müller, N. G., \& Kleinschmidt, A. (2003). Dynamic interaction of object- and space-based attention in retinotopic visual areas. Journal of Neuroscience, 23, 9812-9816.

O'Craven, K. M., Downing, P. E., \& Kanwisher, N. (1999). fMRI evidence for objects as the units of attentional selection. Nature, 401, 584-587.

PALMER, S., \& Rock, I. (1994). Rethinking perceptual organization: The role of uniform connectedness. Psychonomic Bulletin \& Review, 1, 29-55.

Posner, M. I., Snyder, C. R., \& Davidson, B. J. (1980). Attention and the detection of signals. Journal of Experimental Psychology: General, 109, 160-174.

Rock, I., \& GutTman, D. (1981). The effect of inattention on form perception. Journal of Experimental Psychology: Human Perception \& Performance, 7, 275-278.

Serences, J. T., Schwarzbach, J., Courtney, S. M., Golay, X., \&
YANTIS, S. (2004). Control of object-based attention in human cortex. Cerebral Cortex, 14, 1346-1357.

SHAW, M. L. (1978). A capacity allocation model for reaction time. Journal of Experimental Psychology: Human Perception \& Performance, 4, 586-598.

Shomstein, S., \& Behrmann, M. (2006). Cortical systems mediating visual attention to both objects and spatial locations. Proceedings of the National Academy of Sciences, 103, 11387-11392.

Shomstein, S., \& Yantis, S. (2002). Object-based attention: Sensory modulation or priority setting? Perception \& Psychophysics, 64, 41-51.

Shomstein, S., \& Yantis, S. (2004). Configural and contextual prioritization in object-based attention. Psychonomic Bulletin \& Review, 11, 247-253.

Watson, S. E., \& Kramer, A. F. (1999). Object-based visual selective attention and perceptual organization. Perception \& Psychophysics, 61, 31-49.

WolFe, J. M. (1994). Guided Search 2.0: A revised model of visual search. Psychonomic Bulletin \& Review, 1, 202-238.

\section{NOTES}

1. Although here and elsewhere, we discuss attentional prioritization as an ordering of the search, a strict search need not be chosen; an equally plausible implementation would be display-wide parallel processing with different rates of information extraction according to attentional priority.

2. Müller and Kleinschmidt (2003) offered neuroimaging evidence for both prioritization and sensory enhancement accounts of object-based attentional selection. In particular, the authors argued that sensory enhancement accounted for the observation of modulations in the differentobject locations. However, modulations of early sensory regions representing different-object locations could arise from either early sensory enhancement or configural prioritizations.

(Manuscript received January 13, 2006; revision accepted for publication April 15, 2007.) 